\title{
Importaciones efesias en CÁdiz: nuevos hallazgos de la Punta del Nao (La CALETa)
}

Ephesian Imports in Cadiz: New Finds from Punta del Nao (La Caleta)

HORACIO GONZÁLEZ CESTEROS ${ }^{1}$, AURORA HIGUERAS-MILENA CASTELLANO ${ }^{2}$, ANTONIO SÁEZ ROMERO ${ }^{3}$

(1) Österreichisches Archäologisches Institut.hgonces@hotmail.de

(2) Junta de Andalucía. aurora.h.castellano@juntadeandalucia.es

(3) Universidad de Sevilla. asaez1@us.es

\section{RESUMEN:}

Recientes prospecciones subacuáticas, llevadas a cabo en la zona de La Caleta de Cádiz entre 2008 y 2010, han sacado a la luz interesantes materiales relacionados con las distintas fases de la historia de la Bahía de Cádiz. Dentro del estudio general de dichos materiales, este artículo presenta unas pequeñas ánforas producidas en el hinterland de Éfeso, que constituyen las primeras muestras conocidas hasta ahora de este tipo de importaciones en la ciudad de Cádiz. Dichas ánforas pueden considerarse un indicio representativo del comercio de larga distancia y del consumo de productos de alta gama por parte de las élites gaditanas durante el periodo imperial romano y la tardía Antigüedad. En relación a la determinación de estas pautas de consumo se atenderá a la elaboración de productos agrícolas en Éfeso, principalmente vino, y a las estructuras productivas y comerciales de la metrópolis egea.

Palabras clave: Cádiz, ánfora, arqueología subacuática, Éfeso, vino pramnio, Mediterráneo Occidental, Egeo.

\section{Abstract:}

Recent underwater archaeological surveys (2008-2010) carried out in La Caleta area brought to light interesting material from different historical phases in the Bay of Cadiz. Some small amphorae produced in the hinterland of Ephesus are studied in this paper, all of them found in La Caleta, as first evidence of imports from the Aegean metropolis in Cadiz. Those Ephesian pieces could be a representative example of the long distance commerce and the high quality products consumed by the Gaditan elites during the Roman period and the Late Antiquity. To examen in detail this trade we will also refer to the Ephesian agricultural production, particularly wine, and the production and commercial structures of the Aegean metropolis.

Key words: Cadiz, amphorae, underwater archaeology, Ephesus, Pramnian wine, Western Mediterranean, Aegean sea 


\section{UN HALLAZGO EN CONTEXTO SUBACUÁTICO}

Durante los años 2008 a 2010, en el marco del proyecto Aplicación de técnicas geofísicas para la localización, investigación y difusión del Patrimonio Arqueológico Subacuático en la zona de La Caleta (Cádiz), se desarrollaron prospecciones geofísicas y arqueológicas en el entorno subacuático de La Caleta, zona portuaria de gran importancia en la Antigüedad, caracterizada por fondos de poca profundidad ubicados junto al extremo septentrional de la isla menor (la denominada Erytheia o Isla de Juno por los autores clásicos). Debido a su función como puerta principal de entrada a la ciudad fenicia de Gadir y la posterior Gades romana, este canal portuario de La Caleta constituyó, junto a las áreas de fondeadero anexas, un escenario vital para el intenso tráfico marítimo de dichos periodos. Esto dio lugar a que en sus fondos se depositaran gran cantidad de pecios y materiales aislados dispersos. Estas deposiciones fueron documentadas en el curso de las citadas prospecciones, que también incluyeron sondeos selectivos en algunas zonas especialmente significativas, así como el posicionamiento detallado de todos los hallazgos, mayoritariamente ánforas y otras cerámicas que pueden datarse entre los inicios de la presencia fenicia y la Antigüedad Tardía (Higueras-Milena y Sáez 2014).

La erosión marina continuada y la acción antrópica (canteras de piedra, entre otros) durante los últimos tres milenios han modificado notablemente la fisonomía general de La Caleta, y no son escasas las noticias relativas a la desaparición bajo las aguas de cetariae romanas u otros restos de edificaciones de época antigua tanto en el brazo correspondiente al Castillo de San Sebastián como en el entorno del Castillo de Santa Catalina y la Punta del Nao. Es posible, además, que junto a estas instalaciones industriales de época romana, esta segunda área $\mathrm{N}$ de $\mathrm{La}$ Caleta contara con la presencia de algún tipo de santuario dedicado a Astarté/Venus, del cual por el momento no se tiene otra constancia arqueológica que una destacada concentración de piezas identificadas como exvotos o material litúrgico sacadas de los fondos colindantes al bajo de la Punta del Nao (Muñoz 1993; Corzo 1999). En cualquier caso, no cabe duda de que La Caleta constituyó durante todas las etapas de vida de Gadir/Gades, desde su fundación fenicia hasta su decaimiento en época tardoantigua, un punto portuario principal para el asentamiento insular y, por tanto, una zona generadora de gran cantidad de información para la lectura de las pautas comerciales y de consumo de la ciudad.
Las actividades de prospección llevadas a cabo entre 2008 y 2010 comportaron esencialmente el desarrollo de un completo reconocimiento y el cartografiado de anomalías en los puntos principales del entorno de La Caleta, aunque solamente se efectuaron sondeos arqueológicos en puntos concretos en los cuales la concentración de materiales o su especificidad lo requerían. El conjunto de actividades desarrolladas no incluyó por tanto una actuación sistemática y en amplitud sobre ningún pecio de época antigua aunque, no obstante, posibilitó la re-localización de un posible naufragio o cúmulo de naufragios en el cantil $\mathrm{N}$ del brazo rocoso sobre el que se asienta el Castillo de San Sebastián, con materiales locales (Dr. 7/11) e importados (abundantes Dr. 20 y H.70) que sugieren una cronología dentro del s. I d.C. Ningún otro pecio de esta época, o de momentos tardorromanos o tardoantiguos, pudo ser identificado con claridad en este entorno (en una situación similar a otros hallazgos del extremo $\mathrm{S}$ insular, en Sancti Petri, datados en el s. V d.C.; Gallardo et al. 1994; López de la Orden et al. 2001), aunque sí fue posible documentar materiales de estas cronologías dispersos por diversos puntos del canal y de los bajos exteriores de La Caleta.

En concreto, en relación al tipo de materiales que ahora nos ocupa, cabe destacar la recuperación de un asa entera con parte del cuello y la parte superior de la panza de un ánfora de las denominadas one handle Jars, de la forma F 65/66 del Ágora de Atenas (Robinson 1959; Bezeczky 2013), a la que se unirían dos pequeñas ánforas encontradas durante la campaña de prospección del año 2009 en el extremo occidental de la Punta del Nao (en una ubicación cercana entre sí unos $115 \mathrm{~m}$ ) que pueden ser clasificadas sin ningún lugar a dudas como Late Roman Amphora 3 (LRA 3) siguiendo la clasificación de las ánforas tardorromanas y de la Antigüedad Tardía establecida por J. A. Riley en su estudio sobre el material de Cartago (Riley 1981).

El primer ejemplar (CEPE/CA09/115) es indudablemente de época augustea o tiberiana, ya que la misma forma del asa y su enganche con la parte superior de la panza delatan que se trata del tipo más temprano dentro del repertorio de one handle Jars. Las distintas variantes de estos envases fueron clasificadas de manera cronológica por H. S. Robinson en su estudio de la cerámica romana y tardoantigua del Ágora de Atenas (Robinson 1959), siendo producidas desde época augustea hasta el s. IV d.C. Esta pieza se encuentra relativamente erosionada 
Fig. 1: Cartografía de la zona de La Caleta con indicación de los principales hitos y la localización de los tres ejemplares de ánforas efesias estudiados: ánfora del tipo F. 65/66 (1); ánfora LRA3 PNAO/CA09/001 (2) y ánfora LRA3 PNAO/ CA09/067 (3) (sobre la base de Proyección de Mercator del IHM, Cádiz, 1973).

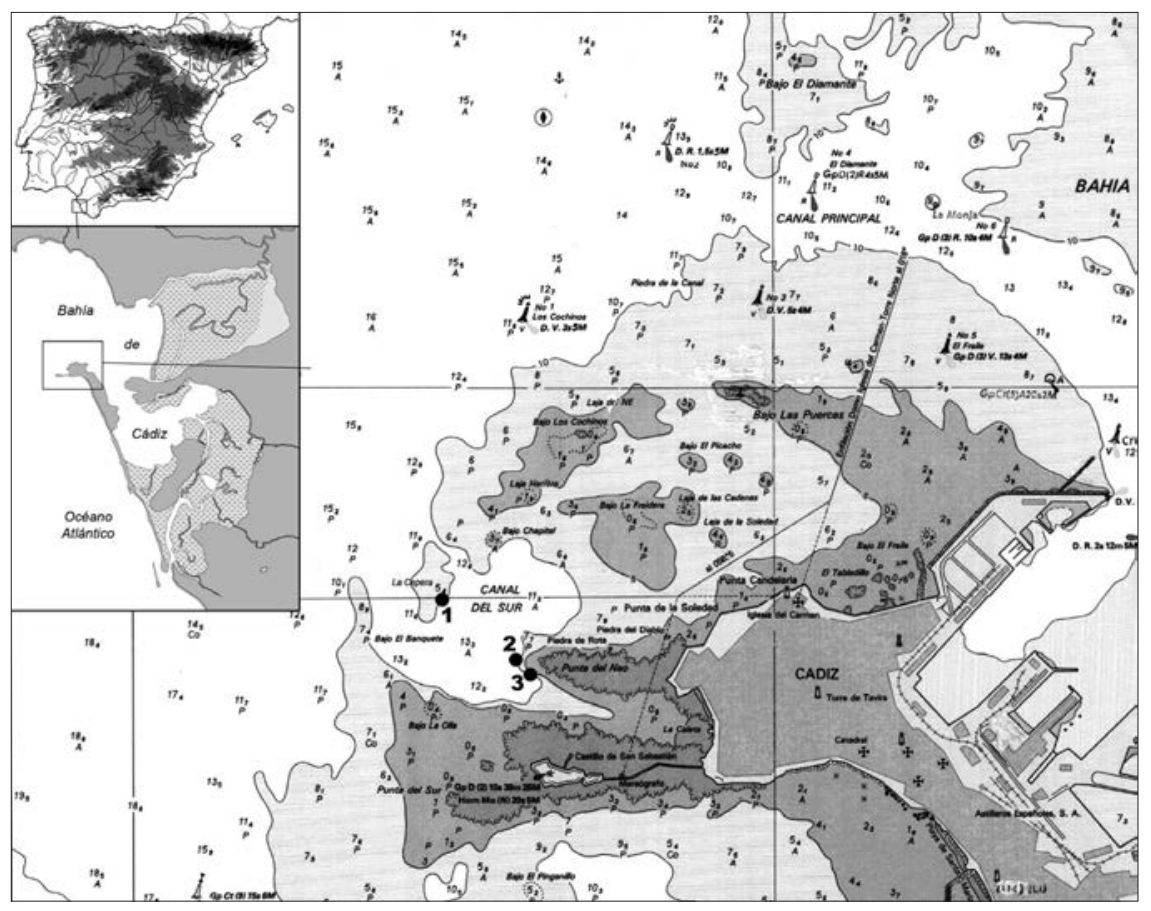

y presenta una pátina oscura debida al hecho de haberse encontrado en un medio subacuático. A pesar de ello, la pasta parece muy similar a algunas de las que conocemos para las producciones efesias de época helenística y romana inicial, siendo bastante cercana a la que T. Bezeczky engloba dentro de su grupo C (Bezeczky 2013: 29-31), que son más calcáreas, con menos inclusiones micáceas y de coloración más clara que las pastas más comunes de Éfeso, como las que presentan los otros ejemplares a los que hacemos referencia en este artículo.

En el caso de las LRA 3, se trata de dos piezas (PNAO/CA-09/067 y PNAO/CA-09/001) que pueden ser consideradas como prototípicas de esta difundida forma de ánfora, especialmente la que presentamos en la fig. 3 ( ${ }^{\circ}$ 067), al contar con un cuerpo fuselado y acanalado, que acaba en un cuello estrecho con dos pequeñas asas y un pequeño borde engrosado. A pesar de que últimamente han sido documentadas algunas imitaciones, siendo el caso más claro las encontradas en los talleres de Cos (Didioumi 2014), el origen y principal lugar de producción se ha de localizar en el hinterland de Éfeso (O de la actual Turquía), algo que concuerda perfectamente con la pasta que presentan ambos ejemplares, de color marrón oscuro, ligeramente rojizo, de superficie con tacto jabonoso, exfoliada en su fractura y con abundantes inclusiones de pequeño tamaño de mica dorada. Dichas características apuntan a su manufactura en los talleres cercanos a la capital asiática (Bezeczky 2013: 28-31).

Dada la ausencia de asociación a restos de un naufragio, e incluso de vinculación espacial directa entre los dos envases, resulta complicado profundizar demasiado en las causas de su deposición en este entorno de Punta del Nao. Es posible especular con la perspectiva de que se trate de evidencias asociadas a algún cargamento de un pecio por ahora no identificado situado en las cercanías. También es factible, sin embargo, que puedan ser ánforas desechadas por la tripulación de buques fondeados entre los ss. V-VI d.C. en el canal portuario de La Caleta una vez vacíos, quedando entonces sin utilidad o posibilidad de reutilización. Tampoco habría que descartar otras opciones menos probables aunque igualmente sugerentes: que sean elementos arrojados a aguas de La Caleta con una función ritual, ligados a la inmemorial tradición cultual de la zona o a ceremoniales marineros; o incluso, que se trate de elementos que habrían podido llegar a contextos subacuáticos debido a la erosión de este frente marítimo. No obstante, esta última opción parece muy poco probable dado el buen estado de conservación de ambos ejemplares. 


\section{IMPORTACIONES EFESIAS EN CÁDIZ Y OTROS LUGARES DEL CÍRCULO DEL ESTRECHO}

En los siguientes párrafos vamos a presentar una breve imagen de las producciones efesias documentadas en la zona del "Círculo del Estrecho", y a ponerlas en relación con la llegada de estos productos a otras regiones del Mediterráneo Occidental. Esto nos permitirá demostrar que la aparición de tres pequeñas ánforas efesias no es sorprendente, ya que si bien son las primeras ánforas de este tipo de las que actualmente se tiene constancia en Cádiz, existe una extensa nómina de lugares donde las one handle Jars y las LRA 3 están documentadas.

En el Mediterráneo Occidental su difusión es notable. En el caso de los envases monoansados de época imperial, dicho mapa de difusión cubre ambas vertientes de la península Itálica, documentándose sobre todo en el entorno de Roma y Pompeya (Panella 1986; Rizzo 2003; Bezeczky 2014) y en el S de Galia (Lemaître 1997; 2002: 220), si bien es cierto que en la mayor parte de las ocasiones se trata de ejemplares algo más tardíos que nuestra pieza de la Caleta, principalmente de los ss. II y III d.C. En este momento, los envases fueron imitados en otros lugares de Asia Menor y el Egeo, como los territorios de Magnesia en el Meandro (Vapur 2009), Pérgamo (Jaap 2014), Sardis (Rauthman 1996) o en Creta (Yangaki 2004/2005), por citar los lugares más importantes. A pesar de la abundante difusión de los envases de época imperial, fue en época tardoantigua cuando las producciones efesias alcanzaron una difusión aún más amplia, como bien pone de manifiesto su elevada presencia en contextos del s. V e inicios del VI d.C., en Roma (Whitehouse et al. 1982; Panella et al. 2010), en Marsella y otros lugares del S de Galia (Bonifay y Pieri 1995; Bonifay et al. 1998; Pieri 1998; 2005).

En la península Ibérica, tanto las LRA 3 como los precedentes monoansados aparecen frecuentemente, sobre todo en puntos del levante y S peninsular. Sin embargo, igual que ocurre en todo el Occidente del Mediterráneo, es realmente en contextos del s. V e inicios del VI d.C. cuando son importadas en cantidades elevadas (Reynolds 1995; Remolà 2000; Morais 2005; Amores et al. 2007; Bernal 2007; García Vargas 2011; etc.), llegando incluso a estar presentes en puntos del Atlántico, sobre todo en Gallaecia (Morais 2005; González Cesteros 2011; Fernández Fernández 2014), así como en importantes centros del interior peninsular como Toledo (Caballero y Sánchez Peláez e.p. ${ }^{1}$.
En yacimientos del denominado "Círculo del Estrecho" están documentadas algunas LRA 3 en lugares como Carteia, Traducta, y Baelo Claudia (Expósito y Bernal 2007; Bernal 2007), si bien su número parece ser bastante reducido. Creemos que la aparición de los ejemplares de Cádiz presentados en este estudio es un indicio de que probablemente llegasen LRA 3 a otros lugares, y nos parece incluso más que posible que en un futuro nos encontremos distintas versiones de one handle Jars en contextos de época imperial.

No tenemos constancia de la existencia de otros hallazgos de este tipo de ánforas en contextos de la ciudad de Cádiz ni en ningún otro punto de la bahía gaditana, pero tampoco sería de extrañar que apareciesen más ejemplos, tanto de LRA 3 como de sus predecesores altoimperiales, ya que las piezas encontradas en la Caleta nos dan un abanico temporal muy amplio. La más temprana de ellas (fig. 2) ha de encuadrarse entre las F65 o F66 de Robinson (1959), que fueron producidas con ligeras variaciones formales desde el último cuarto del s. I a.C. hasta mediados del s. I d.C. Por su parte, los ejemplares gaditanos de LRA 3, se corresponden perfectamente con los tipos del s. V e inicios del VI d.C. (Bezeczky 2013), si bien es cierto que resulta especialmente difícil distinguir entre estas variantes y las de momentos posteriores, siendo este un problema que esperamos resolver gracias a los trabajos que actualmente se están llevando a cabo en contextos de época bizantina inicial y media en el yacimiento de Éfeso ${ }^{2}$. En cualquier caso, y en contra de lo que se ha llegado a presuponer (Pieri 2005: 95; Bezeczky 2013: 164), basándonos en nuestra propia experiencia en contextos bizantinos iniciales de Éfeso, creemos que la producción de este tipo de envases no debió de sobrepasar el final del s. VI d.C., momento de cierta desaceleración de la producción anfórica en Asia Menor. Esta desaceleración precedió a la progresiva desaparición, constatable desde la primera mitad del s. VII d.C., de una gran parte de los envases que habían dominado las exportaciones egeas desde finales del s. IV (Vroom 2003; 2005; 2011; Pieri 2005; Attoui 2011; Poblome 2014).

Conviene hacer una breve referencia a lo que debieron de haber sido las rutas que siguieron nuestra Agora F 65/66 y las LRA 3 hasta llegar a la zona portuaria de La Caleta que, en nuestra opinión, fueron similares a pesar de la diferencia cronológica. Descartando una ruta directa entre la Bética y Éfeso debido a la lejanía entre ambos lugares, lo normal sería pensar que los ejemplares de Cádiz 


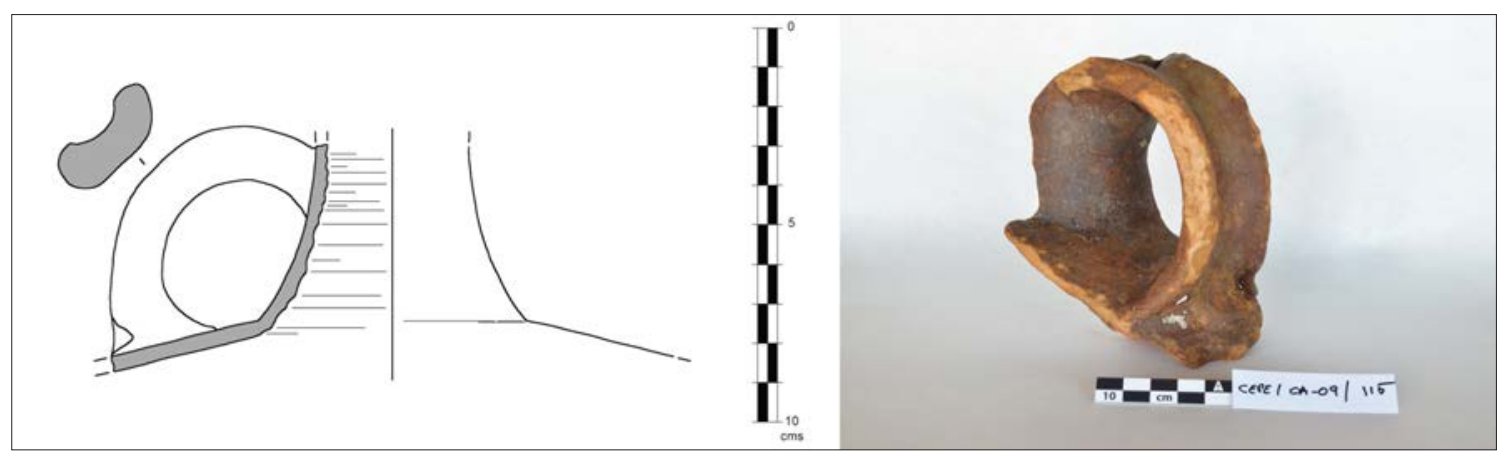

Fig. 2: Ánfora F 65/66 (CEPE/CA09/115).

hubiesen llegado a través de varias escalas, habiendo sido almacenados primero en algún puerto importante del Mediterráneo central, principalmente Roma o Cartago, donde estas formas y otros productos efesios son abundantes (Whitehouse et al. 1982; 1985; Saguì 1998; Panella et al . 2010; Fulford y Peacock 1984). Aparte de las sugerencias sobre Cartago y Roma, resulta imposible precisar otras escalas en dicho trayecto, tanto en el mismo ámbito oriental, donde Creta y Corinto son los puntos mejor posicionados, como en algún puerto hispano meridional, donde Cartagena y Málaga son los centros desde los que con mayor probabilidad se enviaron los productos hacia Gades, que en principio parece haber sido su punto final de consumo.

\section{EL VINO EFESIO Y EL COMERCIO DESDE EL PUERTO DE ÉFESO EN ÉPOCA TARDOANTIGUA}

No es nuestra intención llevar a cabo un estudio exhaustivo acerca de este extenso tema, que sin duda alguna requiere de publicaciones monográficas como la recientemente realizada por T. Bezeczky (2013) sobre las ánforas del periodo romano de la capital asiática. Nuestro objetivo consiste más bien en poner en contexto los ejemplares de la Caleta en relación al tipo de producto que transportaron, la tradición anfórica del lugar de fabricación y el tipo de comercio del que fueron objeto.

La manufactura y exportación masiva de las versiones monoansadas que comienzan a producirse desde época augustea, precisamente con el tipo F 65/66 del ágora de Atenas y de las distintas variantes de LRA 3 de finales del s. IV y finales del VI, es por sí misma plenamente indicativa del amplio comercio de productos líquidos o semilíquidos que se efectuó desde el puerto de Éfeso.
Durante toda la Antigüedad se conoce, a lo largo de la geografía de Asia Menor, la elaboración de distintos bienes susceptibles de ser envasados en ánforas (destacando el vino y el aceite de oliva), tal y como transmiten las numerosas menciones que encontramos en las fuentes escritas desde época clásica hasta el periodo bizantino, pero también las evidencias arqueológicas. Esto queda puesto de manifiesto en la existencia de numerosas prensas utilizadas para la producción de vino y aceite (Aydinoglu y Senol 2010) y la producción de ánforas y otros envases cerámicos utilizados para el transporte de estos bienes en prácticamente cada lugar del O de Anatolia.

A pesar de que las excavaciones en el área de la ciudad greco-romana y bizantina de Éfeso son, sin lugar a dudas, un referente arqueológico para todo el Egeo, conviene precisar que aún queda mucho por hacer en lo referente al hinterland de esta ciudad ${ }^{3}$. Por este motivo, aún hoy en día nos encontramos con una ausencia total de hallazgos tanto de instalaciones productivas como de alfares, si bien parece más que posible que una buena parte de los mismos se encontrasen en las inmediaciones de la ciudad, como ponen de manifiesto los hallazgos de ánforas con fallos de cocción encontrados en distintos puntos del extrarradio de Éfeso (Outschar 1993), o los restos constructivos y de dolia encontrados ocasionalmente en el entorno del Caístro (Bezeczky 2013: 26).

Centrándonos en los pequeños envases efesios de época imperial y tardoantigua, cuya capacidad máxima nunca debió superar los 12-13 litros, contándose en época bizantina con versiones que no superan el litro, generalmente se han propuesto varios tipos de productos con más o menos fundamento, destacando el garum, los ungüentos y el aceite (Pieri 2005: 100-101). Consideramos que conviene descartar la hipótesis del garum, basada tal 


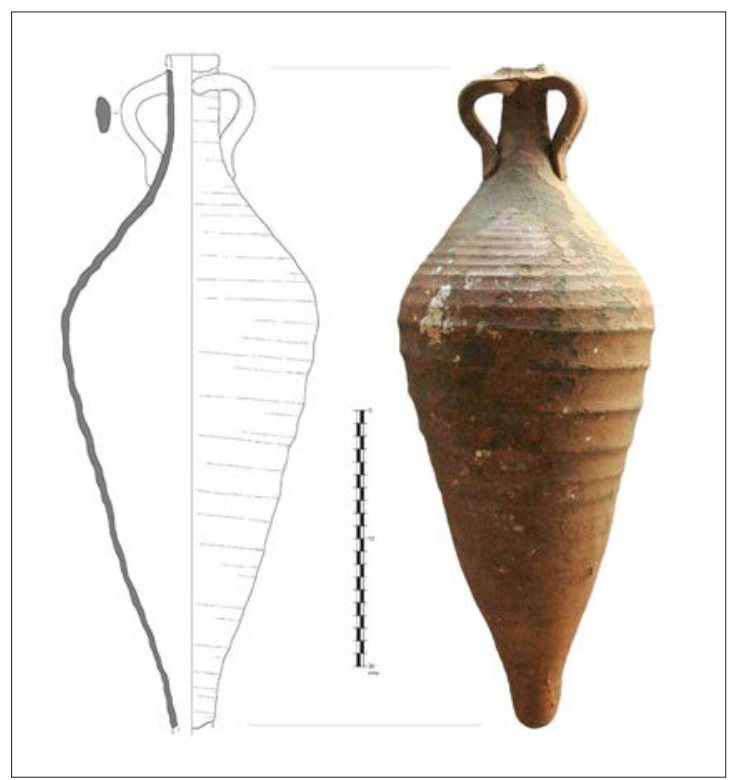

Fig. 3: Ánfora LRA 3 (PNAO/CA09/067).

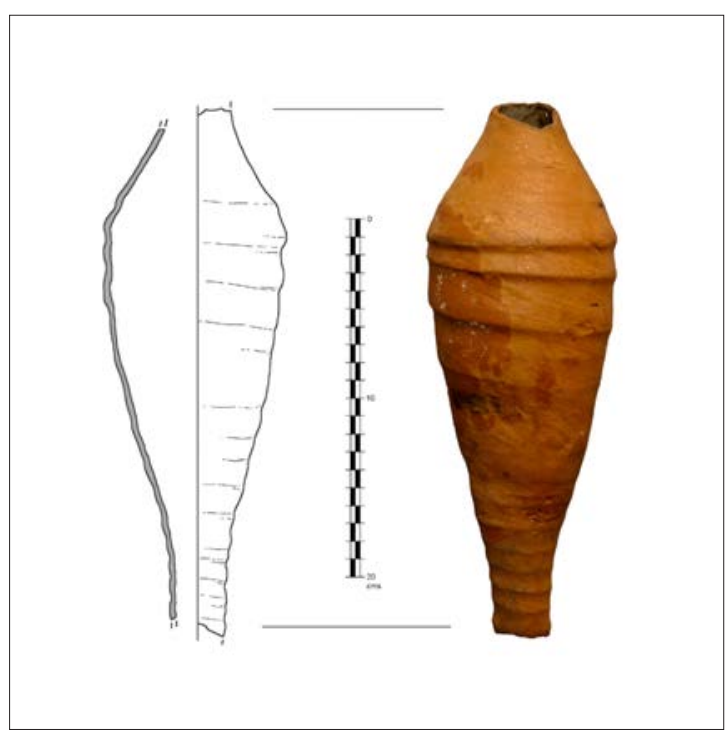

Fig. 4: Ánfora LRA 3 (PNAO/CA09/001).

vez en una cierta similitud de los ejemplares monoansados con los urcei para garum de Pompeya (Cappelletto et al. 2013), ya que ni las fuentes escritas, ni los restos arqueológicos, ni las inscripciones sobre ánforas indican la producción de salsas de pescado en grandes cantidades en el entorno de Éfeso. Sin embargo, en el caso de los ungüentos y/o el aceite, la hipótesis parece estar fundamentada en los análisis de contenido efectuados en un ánfora de la Schola Praeconum de Roma donde se detectaron restos de una sustancia lipídica (Rothschild-Boros 1981). No obstante creemos que conviene ser muy cautos con respecto a los análisis de paleocontenidos, más aún en lo que respecta a los lugares de consumo de las ánforas, pues no hay que descartar un continuo uso secundario de los mismos sobre todo si consideramos que se trata de envases de tamaño y capacidades más o menos reducidas como es el caso de las LRA 3.

Desde nuestro punto de vista, existen varias evidencias que apoyan la hipótesis de que el contenido principal de las one handle Jars y de las LRA 3 fue el vino. Es cierto que los escritores antiguos mencionan la fertilidad del valle del Caístro y de los territorios cercanos, donde se cuenta con todo tipo de productos agrícolas (Bezczky 2013: 25-26), pero también que dentro de las indicaciones de estos escritores destaca el vino por encima de todos. Tanto Estrabón (Geo. XIV, 1, 15) como Plinio (NH 9,75) hablan del vino de Éfeso refiriéndose a él de distinta manera: mientras el primero dice que se trata de un vino de alta calidad, Plinio no lo tiene en muy buena consideración (Bezeczky 2013: 25). Poco después, Ateneo (31d) nos habla del denominado vino pramnio producido en una aldea cercana a Éfeso, y Dioscorides $(5,10)$ lo presenta como un vino efesio. Según Salviat y Tchernia (2013: 126-127) el vino pramnio, mencionado ya en la Iliada (XI, 639), probablemente fue un vino de gran calidad, cuanto menos durante buena parte de la Antigüedad; debió de tener origen jonio y producirse en la provincia romana de Asia y en islas cercanas como Chios, Samos o Icaria, aunque también parece que su vid se cultivó en otros lugares como el Ática ya desde época clásica. Creemos posible que una gran parte de las one handle Jars y las LRA 3 hubiesen servido para envasar y transportar este apreciado vino, ya que el propio tamaño de estos envases sugiere que se trata de exportaciones de productos con un valor bastante alto.

La evidencia epigráfica en soporte pétreo parece respaldar la hipótesis de que el producto envasado fue vino, ya que hace mención a la producción de vino por parte del templo durante época pagana, a la existencia de un collegium de productores de vino en Éfeso y a los impuestos que establecieron los emperadores Valentiniano I, Valente y Graciano sobre la provincia de Asia, que se pagaban principalmente en aceite y vino (Ladstätter 2008: 182). La aparición de algunas muestras epigráficas, principalmente grafitos, sobre ejemplares de ánforas efesias 
Fig. 5: Ungüentaria/anforiscos efesios. Izquierda (Lochner, Sauer y Linke 2003). Derecha foto González Cesteros. Depósito del Instituto Austriaco de Arqueología en Selçuk.

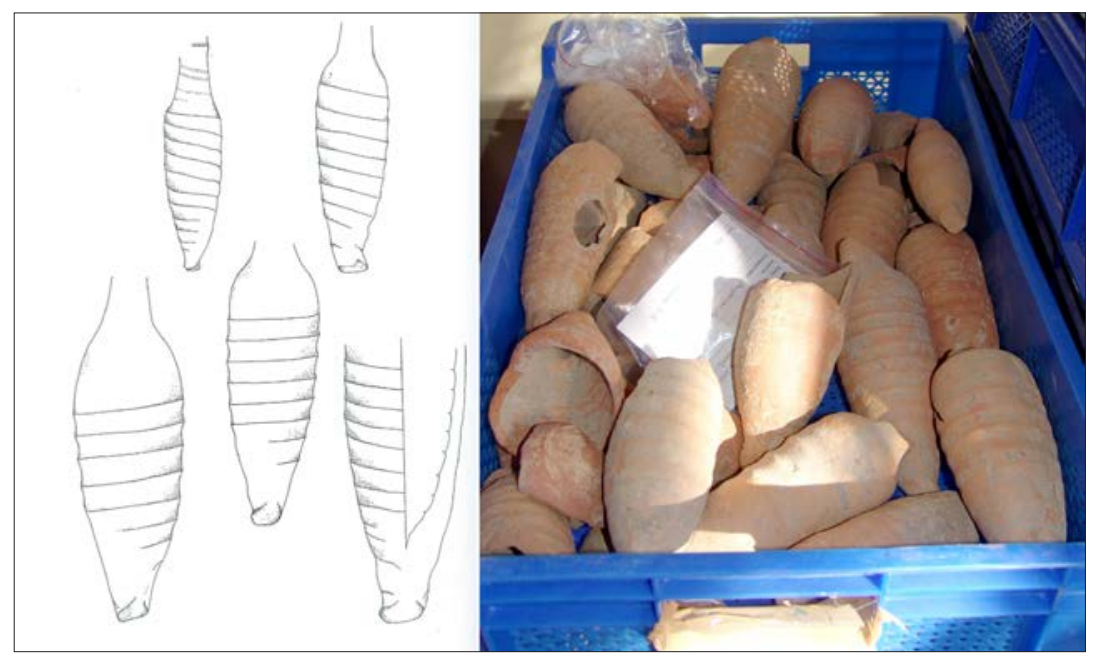

en los que se hace mención a un tipo de vino (Ladstätter 2008: 181; Ladstätter y Pülz 2008: 423-424; Pieri 2005: 101), ofrece una evidencia más que refuerza dicha hipótesis vinaria.

Otro posible elemento que en teoría podría ayudarnos a decantarnos por un contenido vinario sería la presencia continua de resina en las paredes interiores de estos envases, especialmente en LRA 3, algo que también ocurre en el ejemplar de la fig. 3 (PNAO/CA-09/067) encontrado en la Punta del Nao. Sin embargo, en los últimos años se ha puesto de manifiesto que el uso de esta sustancia para impermeabilización también debió de ser común en el caso de distintos tipos de salsas de pescado e incluso del aceite de oliva (Garnier et al. 2011: 410).

Cabe mencionar que, a pesar de ser las exportaciones más difundidas, las one handle Jars y las LRA 3 no fueron los únicos tipos de ánforas que se fabricaron en el territorio de Éfeso. Como ya se ha señalado, hasta la fecha no existe ningún alfar que haya sido claramente documentado en las cercanías de la ciudad, pero ello no impide el claro reconocimiento de una producción local con pastas bien caracterizadas y que cuenta con el respaldo de estudios arqueométricos que han conseguido que pueda ser perfectamente individualizada ${ }^{4}$. Partiendo de esta base, actualmente se ha podido documentar para el periodo helenístico y augusteo la existencia de un variado repertorio de ánforas efesias (Lawall 2004; Bezeczky 2013), entre las que cabe destacar la producción ocasional de ejemplares de tipo rodio y de Dressel 24 iniciales a comienzos de la época romana. En el s. III d.C. también se cuenta con Kapitän 2 con pastas efesias, aunque curiosamente no se ha encontrado ninguna en el propio yacimiento (Bezeczky 2013) ${ }^{5}$. Desde los trabajos realizados en la Necrópolis del Puerto, también se ha puesto de manifiesto una producción de ánforas de tipos Cos y de G. 198 entre los ss. I y III d.C. (González Cesteros y Yilmaz e.p.).

Sin embargo, creemos que, tal vez a excepción de las Dressel 24 y de las ánforas tipo Cos, el resto de producciones debieron de ser totalmente minoritarias e incluso esporádicas, siendo de nuevo en época tardoantigua cuando se constata la producción en gran volumen de otros tipos de ánforas, probablemente al amparo de la difusión alcanzada por las LRA 3. En este sentido, habría que incluir a una gran parte de las ánforas de lo que deberíamos denominar Samos Cistern Family, en la cual incluimos a las M. 273, a las propias Samos Cistern type y a otros tipos semejantes, que son muy abundantes en contextos efesios que presentan grandes cantidades de material datable en los ss. V y VI d.C., destacando una vez más la Necrópolis del Puerto (González Cesteros y Yilmaz e.p.). Entre estos tipos existe un alto porcentaje cuyas pastas son idénticas a las de las producciones efesias, por lo que parece más que probada una producción en Éfeso o en sus inmediaciones junto con otros lugares del E del Egeo.

Otro tipo de ánfora plenamente efesia producida durante el periodo bizantino inicial son las denominadas Ephesos 56. Se trata de un ánfora de forma oval y pequeño tamaño, que generalmente no supera los 60 centímetros de altura, con una boca y cuellos muy estrechos, y que fue identificada por primera vez como una producción efesia 


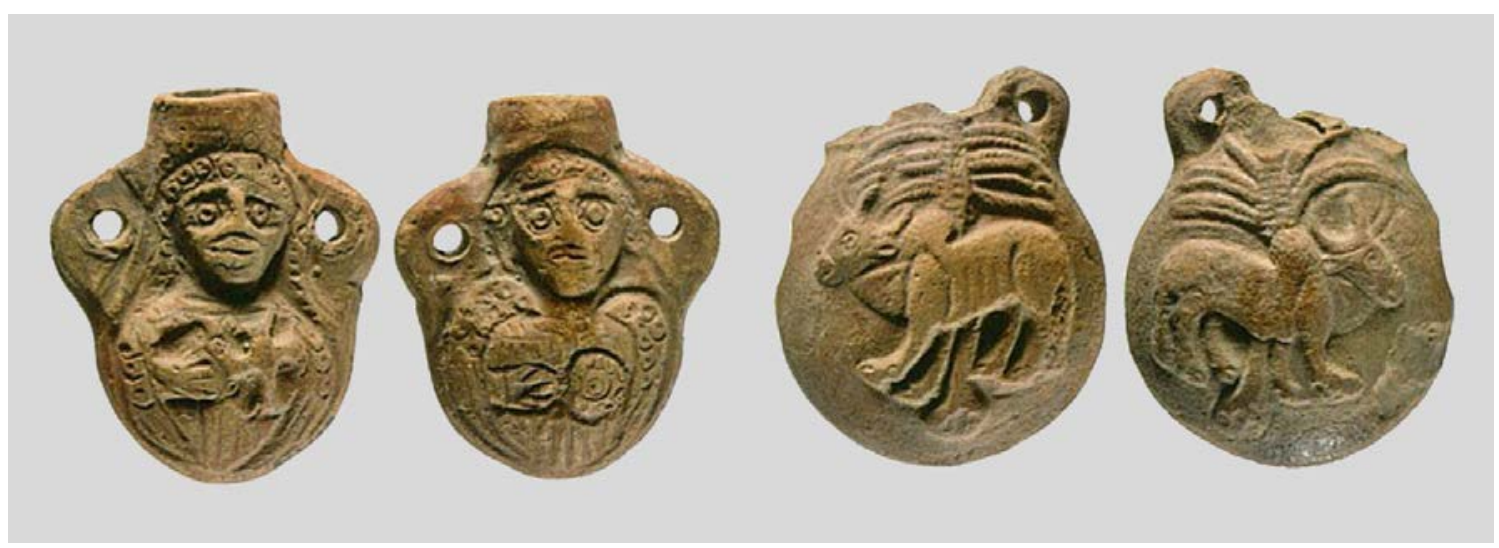

Fig. 6: Pilgernflächchen (Ladstätter 2010).

por T. Bezeczky a partir de los hallazgos del Ágora de Tetrágonos en Éfeso (Bezeczky 2005: 204). En cuanto al margen cronológico en el que fue producida, cabe señalar que probablemente abarcase desde finales del s. V d.C. hasta finales del s. VI ó inicios del VII d.C., estando presente en grandes cantidades en contextos efesios del s. VI (Ladstätter 2008: 182; Bezeczky 2013: 169). Se ha planteado tímidamente que este ánfora pudo servir para envasar aceite de oliva (Bezeczky 2013: 168), algo que creemos posible al presentar un cuerpo ovalado y convivir con las LRA 3, que son ánforas eminentemente vinarias (vide supra). Esto implicaría una dualidad de productos exportados desde Éfeso en la Antigüedad tardía, que podría tener su correspondiente en época altoimperial con las one handle Jars y las Dressel 24, aunque hay que admitir que la producción de este último tipo en Éfeso no parece haber alcanzado el nivel de las Ephesos 56. La difusión de las Ephesos 56 más allá del ámbito egeo está bien documentada a lo largo del s. VI d.C. (Bezeczky 2013: 168; González Cesteros e.p.), aunque hasta la fecha no la tenemos localizada en la península Ibérica.

Junto con la producción de grandes cantidades de ánforas, hay que mencionar la producción y exportación de otros tipos de envases cerámicos desde el puerto de Éfeso durante los ss. V y en menor medida el VI d.C. Se trata de algunos de los denominados Late Roman Unguentaria, principalmente de la forma B de Riley (1975), que es típicamente efesia, pero también de imitaciones locales de la forma A de Riley (Metaxas 2005: 95-97), y de los 1lamados Ephesian early Byzantine Amphoriskos. Incluso en el actual estado embrionario de la investigación, cuando los alfares que produjeron estos pequeños envases todavía no han sido localizados, el examen de las pastas permite confirmar que se hicieron en los mismos talleres que las LRA 3 (Löchner et al. 2005; Metaxas 2005). Sin lugar a dudas fueron exportados en cantidades ingentes, como demuestran tanto su elevada presencia en las excavaciones y prospecciones en Éfeso, como su relativamente alta difusión (Metaxas 2005: 98) ${ }^{6}$, más fácil de seguir para el caso de los Ephesian early Byzantine Amphoriskos al tratarse de una producción cuyos rasgos formales pueden ser diferenciados de los unguentaria.

Igualmente, para entender el comercio de Éfeso a lo largo de toda su historia, pero especialmente en época bizantina inicial, hay que tener en cuenta la difusión de unos pequeños envases cerámicos, generalmente de no más de ocho centímetros de altura y cinco de anchura, que actúan como pequeñas cantimploras o botellitas (fig. 6, Ladstätter y Pülz 2008: 428; Ladstätter 2010: 507-508). Estos recipientes, denominados Pilgernfläschchen o Pilgerampullen en la bibliografía de habla alemana relacionada con Éfeso, funcionarían del mismo modo que las ampullae de San Menas, es decir, serían Eulogia para los peregrinos, formando parte de un tipo de comercio plenamente religioso, en este caso de peregrinación, que parece tener un papel importantísimo en las ciudades de Asia Menor desde el inicio de la colonización griega, y que sin lugar a dudas tiene en Éfeso a uno de los ejemplos más notorios en época romana y bizantina (Pülz 2010; Külzer 2010).

Resulta evidente que en el caso de las ánforas no podemos hablar de un comercio relacionado con la peregrinación religiosa, menos aún cuando las LRA 3 siguen unas rutas de difusión muy similares a las que ya tuvieron las ánforas monoansadas de época alto y medio imperial. 
Sin embargo, consideramos que está fuera de cualquier duda que la Iglesia desempeñó un importante papel dentro de la producción y comercio a gran escala a partir del s. V d.C., sobre todo a partir del momento en que pasó a estar vinculada directamente con el estado y a formar parte de su administración (Durliat 1998; Bernal 2010). En este sentido, resulta sintomático que sea a partir del s. V d.C. cuando se observe la mayor difusión de los productos efesios, principalmente de las LRA 3, pues se trata de un momento de auge tanto para la ciudad de Éfeso como para su Iglesia (Ladstätter 2010; Pülz 2010), pero también de cambios de propiedad, al dejar de funcionar todo el sistema de producción de época anterior, que tenía como pilar fundamental el control de las tierras y la producción por parte del templo de Ártemis, que ahora pasarían a estar en manos estatales y eclesiásticas (Ladstätter y Pülz 2008: 422-423) .

\section{CONCLUSIONES}

En estas páginas hemos intentado centrarnos en dos aspectos novedosos para los estudios arqueológicos de época romana y tardoantigua en la península Ibérica. El primero es el hallazgo en el entorno de La Caleta de unos ejemplares de ánforas producidas en el hinterland de Éfeso, que no habían sido documentadas con anterioridad en Gades.

Hasta la fecha estaban documentadas otras importaciones del Mediterráneo Oriental en Gades donde, destacando para el periodo tardoantiguo ánforas de procedencia Sirio-anatólica, como las LRA 1 (Expósito y Bernal 2007). Al poner a Cádiz en el mapa de difusión de LRA 3 y de sus predecesoras monoansadas de época imperial, se aporta un nuevo argumento para poder explicar la presencia de importaciones orientales en puntos más allá de las Columnas de Hércules, si bien creemos que no puede considerarse que todas las importaciones del Mediterráneo Oriental pudieran haber sido enviadas de manera conjunta hasta los puntos de consumo más Occidentales del mundo romano y tardoantiguo.

La presencia de LRA 3 en el ámbito atlántico peninsular está principalmente documentada al $\mathrm{N}$ del Tajo (Morais 2005; Fernández Fernández 2014) ${ }^{8}$, ya que hasta la fecha no se conocen importaciones en el Algarve y Alentejo (Almeida et al. 2014: 155) o en la provincia de Huelva. No obstante, hay que reconocer que aún queda pendiente para futuras fases de investigación desentrañar las claves de la presencia de estas ánforas en la Punta del Nao. En cualquier caso, deben verse como una muestra del consumo de productos de Asia Menor en la ciudad gaditana, cuanto menos durante distintos momentos de la Antigüedad.

Otro factor importante para intentar explicar la presencia de F 65-66 y LRA 3 en Cádiz y que hemos intentado tratar de forma resumida, se refiere a la contextualización de la producción de Asia Menor y principalmente de Éfeso en época romana y tardoantigua, así como al producto que transportaron estos envases. Creemos que hoy en día está fuera de duda que el vino fue el principal contenido que envasaron. Es más, probablemente se trate de un vino de alta gama, tal vez el famoso pramnio al que se refieren las fuentes escritas, cuya difusión fue siempre más o menos regular. Sin embargo, es durante los ss. V y VI d.C., al amparo de la relevancia económica y comercial que adquiere la Iglesia, así como del auge de Éfeso como un centro cristiano de primer nivel dentro del Mediterráneo Oriental, cuando este vino y otros productos efesios van a alcanzar su mayor nivel de exportación.

La llegada de las LRA 3 a Gades ha de enmarcarse dentro de este contexto de auge productivo y comercial de Éfeso. La presencia de las mismas nos indica que si bien hacía tiempo que Gades había perdido la relevancia comercial que tuvo en momentos anteriores, aún debía de contar con instalaciones portuarias (Bernal 2012) y con élites pudientes a las que llegasen bienes de consumo de todo el Mediterráneo, como sugieren diversos hallazgos en el actual casco urbano de El Puerto de Santa María, identificado con Portus Gaditanus, que incluyen materiales de similar cronología a las LRA 3 de La Caleta (Lagóstena et al. 1996; Lagóstena 1998; López Amador y Pérez 2013). Por su parte, el fragmento de F 65-66 constituye una muestra más de la potencia de Gades como puerto comercial de primera relevancia en época Augustea y Julio-Claudia.

\section{NOTAS}

1. Hay que subrayar que la cuestión de las importaciones de ánforas y otros tipos de cerámicas orientales a puntos del interior peninsular durante época tardoantigua continúa sin estar estudiada en profundidad, a pesar de las excavaciones acometidas en lugares como La Vega Baja de Toledo o la villa de Carranque, por citar sólo dos ejemplos destacados.

2. Las excavaciones de contextos de época bizantina inicial y media en la zona portuaria y parte baja de la ciudad de Éfeso están aportando un abundantísimo material cerámico, que aún se encuentra en proceso de estudio. Esperamos que en un futuro no muy distante estemos en disposición de poder presentar interesantes novedades al respecto gracias al proyecto Lise 
Meitner concedido por el Fondo de Investigación Austriaco a uno de nosotros para el estudio de las ánforas bizantinas de varios contextos de Éfeso.

3. La principal publicación sobre el hinterland efesio es el trabajo de R. Meriç (2009) sobre el valle del Caístro, que proporciona una panorámica desde el Neolítico hasta época romana. Sería conveniente que este trabajo de amplia diacronía fuese ampliado y revisado para poder tener una visión más detallada de la explotación del territorio durante la Antigüedad y el periodo bizantino.

4. En este sentido destacan los trabajos llevados a cabo por R Sauer y T. Bezeczky así como en los últimos tiempos por L. Peloscheck.

5. Tanto a este investigador como a nosotros mismos nos ha sido imposible documentar ninguna Kapitän 2 entre las cantidades ingentes de material trabajadas hasta la fecha en Éfeso. No obstante, la presencia de Kapitän 2 en contextos del s. III en Éfeso es tan abrumadoramente alta, que creemos bastante factible que se produjeran imitaciones de esta forma.

6. Estamos convencidos que en un futuro cercano la nómina de lugares de importación se irá ampliando continuamente, ya que aún hoy en día, al no contarse con ejemplares más o menos completos, suelen pasar inadvertidos o son directamente confundidos con aquellos cuya producción no es efesia. Para la presencia de Late Roman Unguentaria en la península Ibérica: Vizcaíno Sánchez 2009, 636-648.

7. Como bien señalan ambos investigadores, la ausencia de fuentes escritas y epigráficas respecto a este tema nos impide poder asegurar esta cuestión, pero los propios cambios urbanísticos y arquitectónicos que se observan en la ciudad de Éfeso, así como en las producciones cerámicas a ella vinculadas, llevarían a abogar por una serie de transformaciones dirigidas directamente por la administración imperial.

\section{BIBLIOGRAFÍA}

ALMEIDA, R.; VIEGAS, C.; BEJA, N.; TEXEIRA, N. (2014): Ânforas do Mediterràneo oriental em Faro (Ossonoba). Novos dados para equacionar o comércio durante a Antiguidade Tardia), As produçoes cerâmicas de imitação na Hispania (R. Morais, A. Fernández, M.J. Sousa, eds.), Monografías Ex Officina Hispana II, Oporto, 151-160.

AMORES, F.; GARCÍA VARGAS, E.; GONZÁLEZ ACUÑA, D. (2007): Ánforas tardoantiguas en Hispalis (Sevilla, España) y el comercio Mediterráneo, Late Roman Coarse Wares 2 (M. Bonifay, J-C. Tréglia, eds.), Oxford, 133-146.

ATTOUI, R. (ed.) (2011): When did Antiquity End? Archaeological case studies in three continents, BAR. Int. Ser. 2268, Oxford.

AYDINOGLU, Ü.; SENOL, A. K. (eds.) (2010): Olive oil and wine production in Anatolia during Antiquity, International Symposium Proceedings, Estambul.

BERNAL, D. (2007): Contextos cerámicos en el área de estrecho de Gibraltar (ss. V-VII d.C.): Hacia el replanteo de la dinámica urbana, económica y comercial tardorromana, Late Roman Coarse Wares 2, Vol. I (M. Bonifay, J-C. Tréglia, eds.), BAR Int. Ser. 2185 (I), Oxford, 109-117.
BERNAL, D. (2010): Iglesia, producción y comercio en el Mediterráneo tardoantiguo. De las ánforas a los talleres eclesiásticos, Late Roman Coarse Wares 3, vol. I (S. Menchelli, S. Santoro, M. Pasquinucci, G. Guiducci, eds.), BAR Int. Ser. 2185 (I), Oxford, 19-31.

BERNAL, D. (2012): El puerto romano de Gades: novedades arqueológicas, Rome, Portus and the Mediterranean (S. Keay, ed.), Archaeological Monographs of the British School at Rome 21, Londres, 225-244.

BEZECZKY, T. (2005): Late Roman Amphorae from the Tetragonos Agora in Ephesus (mit einem Beitrag von Peter Scherrer), Spätantike und mittlelalterliche Keramik aus Ephesos (F. Krinzinger, ed.), Denkschriften der philosophisch-historischen Klasse 332, Archäologische Forschungen 13, Viena, 203-229.

BEZECZKY, T. (2013): The amphorae of Roman Ephesus, Forschungen in Ephesos XV/1.

BONIFAY, M.; PIERI, D. (1995): Amphores du Ve au VIIe s. à Marseille: nouvelles données sur la typologie et le contenu, Journal of Roman Archaeology 8, 94-120. DOI: https://doi.org/10.1017/S1047759400015993

BONIFAY, M.; CARRE, M.-B.; RIGOIR, Y. (1998): Fouilles à Marseille. Les mobiliers (Ier-VIIe siècles ap. J.-C.), Travaux du Centre Camille-Jullian 22.

BRUN, J.-P. (2013): Los usos antiguos de los productos de la viña y el olivo y sus implicaciones arqueológicas, De vino et oleo Hispaniae (J. M. Noguera, J. A. Antolinos, eds.), Murcia, 19-35.

CABALLERO, R.; SÁNCHEZ PELÁEZ, I. (e.p.): La tardoantigüedad en Toledo reflejada en las ánforas recuperadas en la $\mathrm{C} /$ Cuesta de los Portugueses, III Congreso Internacional de la SECAH (Tarragona 2014).

CAPELLETTO, E.; BERNAL, D.; COTTICA, D.; BUSTAMANTE, M.; LARA, M.; SÁEZ, A. M. (2013): Urcei per salse di pesce da Pompei-Ercolano: una prima análisis, Hornos, talleres y focos de producción alfarera en Hispania. I Congreso Internacional de la SECAH Ex Officina Hispana, tomo II, (D. Bernal, L. C. Juan, M. Bustamante, J. J. Díaz, A. M. Sáez, coords.), Madrid, 271-280.

CORZO, R. (1999): Venus Marina Gaditana, Fundación El Monte, Sevilla.

DIADUMI, S. (2014): Local pottery production in the island of Cos, Greece from the early Byzantine period. A preliminary report, Late Roman Coarse Wares 4 (N. Poulou-Papadimitriou, E. Nodarou, V. Kilikoglou, eds.), BAR. Int. Ser. 2616, I, Oxford, 169-180.

DURLIAT, J. (1998): Les conditions du commerce au VIe siècle, The sixth century. Production, Distribution and Demand (R. Hodges, W. Bowden, eds.), Leiden-Boston-Colonia, 89-117.

EXPÓSITO, J. A.; BERNAL, D. (2007): Ánforas orientales en el extremo Occidente: Las importaciones de LR 1 en el sur de Hispania, Late Roman Coarse Wares 2 (M. Bonifay, J-C. Tréglia, eds.), BAR. Int. Ser. 2185, I., Oxford, 119-132. 
FERNÁNDEZ FERNÁNDEZ, A. (2014): El comercio tardoantiguo (ss. IV-VII) en el Noroeste peninsular a través del registro cerámico de la ría de Vigo, Roman and Late Antique Mediterranean Pottery 5, Oxford.

FULFORD, M. G.; PEACOCK, D. P. S. (1984): Excavations at Carthage: The British Mission I 2. The Avenue du president Habib Bourguiba, Salammbo: The Pottery and Other Ceramic Objects from the Site, Sheffield.

GALLARDO, M.; MARTÍ, J.; ALONSO, C.; GARCÍA, C. (1994): Prospecciones arqueológicas subacuáticas en Sancti-Petri, AAA 1994, Tomo II, 44-48.

GARCÍA VARGAS, E. (2011): Oriental trade in the Iberian Peninsula during Late Antiquity (4th-7th centurias AD): An Archaeological Perspective, New Perspectives on Late Antiquity (D. Hernández de la Fuente, ed.), Cambridge, 76-117.

GARNIER, N.; SILVINO, T.; BERNAL, D. (2011): L'identification du contenu des amphores: huile, conserves de poissons et poissage, Actes de la SFECAG, Congrès d'Arles, Marsella, 397-416.

GONZÁLEZ CESTEROS, H. (2011): Las ánforas orientales de Lugo, Ánforas romanas de Lugo. Comercio romano en el Finis Terrae (Carreras, R. Morais, eds.) Traballos de Arqueoloxía, 3, Lugo, 108-127.

GONZÁLEZ CESTEROS, H. (e.p.): Eastern Mediterranean Amphorae, Amphora Research in Castrum Villa on Brijuni Island (T. Bezeczky, ed.), Viena.

GONZÁLEZ CESTEROS, H.; YILMAZ, Z. (e.p.): Amphorenfunde aus der Hafennekropole, Forschungen in der Hafennekropole von Ephesos. Die Grabungen seit 2005 im Bereich des Hafenkanals. Sonderschriften des Österreichischen Archäologischen Instituts (M. Steskal, ed.), Viena.

HIGUERAS-MILENA, A.; SÁEZ, A. M. (2014): Aplicación experimental de técnicas geofísicas para la localización, investigación y difusión del patrimonio arqueológico en la zona de La Caleta (Cádiz), Arqueología Subacuática Española. Actas del I Congreso de Arqueología Náutica y Subacuática Española, vol. II (X. Nieto, M. Bethencourt, eds.), Cádiz, 275-286.

JAAP, S. (2014): Micaceous waterjars in Pergamon - a specific variante of the Late Roman 2 amphora, Late Roman Coarse Wares 4, vol. I (N. Poulou-Papadimitriou, E. Nodarou, V. Kilikoglou, eds.), Oxford, 143-150.

KAUFMANN, C. M. (1910): Zur Ikonographie der Menas-Ampullen, Veröffentlichungen der Frankfurter Menasexpedition, vol. 5, Cairo.

KÜLZER, A. (2010): Handelsgüter und Verkehrswege: Wirtschaftliche Aspekte byzantinischer Pilgerzentren, Handelsgüter und Verkehrswege. Aspekte der Warenversorgung im östlichen Mittelmeerraum (4. Bis 15. Jahrhundert). Veröffentlichungen zur Byzanzforschung, Band XVIII (E. Kislinger, J. Koder, A. Külzer, eds.), Viena, 185-196.
LADSTÄETTER, S. (2008): Römische, spätantike und byzantinische Keramik, Das Vediusgymnasium in Ephesos. Archäologie und Baubefund (Forschungen in Ephesos 14, 1) (M. Steskal, M. la Torre, eds.), Viena, 97-189.

LADSTÄETTER, S. (2010): Ephesos in byzantinischer Zeit. Das letzte Kapitel der Geschichte einer antiken Großstadt, Byzanz-Das Römerreich im Mittelalter II, 2. Schauplätze (F. Daim, J. Drauschke, eds.), Monographien des Römisch-Germanischen Zentralmuseums 84, 2, 2., Mainz, 493-519.

LAGÓSTENA, L. (1998): La bahía gaditana en la Antigüedad Tardía, Homenaje al Dr. Carlos Posac Mon, Ceuta, 265-278.

LAGÓSTENA, L.;TORRES, J.; LAPEÑA, O.(1996): Aproximación a la ocupación tardorromana en la desembocadura del río Guadalete (Puerto de Santa María, Cádiz), AUC XI, 95-122.

LAWALL, M. L. (2004): Archaeological Context and Aegean Amphora Chronologies: A Case Study of Hellenistic Ephesos, Transport Amphorae and Trade in the Eastern Mediterranean (J. Eiring, J. Lund, Eds.), Monographs of the Danish Institute at Athens 5, Aarhus, 171-188.

LEMAÎTRE, S. (1997): L'amphore de type Agora F65/66 dite "Monoansée". Essai de synthèse à partir d'exemplaires lyonnais, SFECAG, Actes du Congrès du Mans, Marsella, 311-320.

LEMAÎTRE, S. (2002): Recherche sur la difusión en Gaule des amphores produites dans le sud-ouest de l'Anatolie à l'époque impériale, Céramiques hellénistiques et romaines. Productions et difusión en Méditerranée orientale (Chypre, Égypte et côte syro-palestinienne) (F. Blondé, P. Ballet, J.-F. Salles, eds.), Lyon, 213-226.

LÖCHNER, S.; SAUER, R.; LINKE, R. (2003): Late Roman Unguentaria? A contribution to early byzantine wares from the view of Ephesus, Late Roman Coarse Wares 1 (M. A. Gurt, J. Buxeda, M. A. Cau, eds.), BAR. Int. Ser. 1340, Oxford, 647-654.

LÓPEZ AMADOR, J. J.; PÉREZ FERNÁNDEZ, E. (2013): $E l$ Puerto Gaditano de los Balbo. El Puerto de Santa María. Cádiz, El Puerto de Santa María.

LÓPEZ DE LA ORDEN, M. D.; GALLARDO, M.; BLANCO, F. J. (2001): Estudio numismático de monedas bajoimperiales procedentes del yacimiento subacuático de Lavaculos (Sancti Petri. Cádiz), Boletín del Instituto Andaluz de Patrimonio Histórico 37, 127-135.

MERIÇ, R. (2009): Das Hinterland von Ephesos. Archäologischtopographische Forschungen im Kaystros Tal, Ergänzungshefte zu den Jahresheften des Österreichischen Archäologischen Institutes in Wien, Heft 12.

METAXAS, S. (2005): Frühbyzantinische Ampullen und Amphoriskoi, Spätantike und mittlelalterliche Keramik aus Ephesos (F. Krinzinger, ed.), Denkschriften der philosophischhistorischen Klasse 332, Archäologische Forschungen 13, Viena, 67-123. 
MORAIS, R. (2005): From Oppidvm to Dives Bracara: The city trade through the amphorae, Late Roman Coarse Wares 1 (M.A. Gurt, J. Buxeda, M. A. Cau, eds.), BAR. Int. Ser. 1340, Oxford, 55-67.

MUÑOZ, A. (1993): Las cerámicas fenicio-púnicas de origen submarino del área de la Caleta (Cádiz), CPAC 15, 287-334.

OUTSCHAR, U. (1993): Produkte aus Ephesos in alle Welt?, Österreichisches Archäologisches Institut. Berichte und Materialien, Heft 5, 47-52.

PANELLA, C. (1986): Oriente ed Occidente: Considerazioni su alcune anfore "egee" di età imperiale a Ostia, Recherches sur les amphores grecques. Actes du Colloque International organisé par le Centre National de la Recherche Scientifique, l'Université de Rennes II et l'École Française d'Athènes (J.-Y. Empereur, Y. Garlan, eds.), BCH Suppl. 13, Atenas, 609-636.

PANELLA, C.; SAGUÌ, L.; CASALINI, M.; COLETTI, F. (2010): Contesti Tardoantichi di Roma: una rilettura alla luce di nuovi dati, Late Roman Coarse Wares 3, Vol. I (S. Menche1li, S. Santoro, M. Pasquinucci, G. Guiducci, eds.), BAR Int. Ser. 2185 (I), Oxford, 57-78.

PIERI, D. (1998): Les importations d'amphores orientales en Gaule méridionale durant 1'Antiquité tardive et le Haut Moyen Âge (IVe-VIIe siècle après J.-C.). Typologie, chronologie et contenu, SFECAG, Actes du Congrès d'Istres, Marsella, 97-106.

PIERI, D. (2005): Le commerce du vin oriental à l'époque byzantine (Ve-VIIe siècles). Le témoignage des amphores en Gaule, Bibliothèque Archéologique et Historique. T. 174, Beirut.

POBLOME, J. (2014): Shifting societal complexity in Byzantine Asia Minor and Dark Age pottery, Late Roman Coarse Wares 4, Vol. I (N. Poulou-Papadimitriou, E. Nodarou, V Kilikoglu eds.), BAR Int. Ser. 2616 (I), Oxford, 623-642.

PÜLZ, A. (2010): Ephesos als christliches Pilgerzentrum, Mitteilungen zur christlichen Archäologie, Band 16, 71-104. DOI: https://doi.org/10.1553/micha16s71

RAUTMAN, M. L. (1996): Two Late Roman Wells at Sardis, Preliminary excavation reports: Sardis, Idalion and Tell ElHandaquq North (W. G. Dever, ed.), AASOR 53, 37-84.

REMOLÀ, J. A. (2000): Las ánforas tardo-antiguas en Tarraco (Hispania Tarraconensis), Col·lecció Instrumenta, 7, Barcelona.

REYNOLDS, P. (1995): Settlement and pottery in the Vinalopó Valley (Alicante, Spain) A.D. 400-700, BAR Int. Ser. 604, Oxford.

REYNOLDS, P. (2005): Hispania and the Late Roman Mediterranean Ceramics and trade, Hispania in Late Antiquity. Current Perspectives, 369-486.
RILEY, J.A. (1975): The pottery from the first session of excavation in the Caesarea hippodrome, Bulletin of the American Schools of Oriental Research 218,25-54.

RILEY, J. A. (1981): The pottery from the cisterns 1977.1, 1977.2 and 1977.3, Excavations at Carthage conducted by the University of Michigan, vol. VI (J.H. Humphrey, ed.), Ann Arbor, 85-124. DOI: https://doi.org/10.2307/1356166

ROBINSON, H. S. (1959): The Athenian Agora V. Pottery of the Roman Period, Chronology, Princeton.

ROTHSCHILD-BOROS, M. C. (1981): The Determination of Amphora Contents, Archaeology and Italian Society (G. Barker, R. Hodges, eds.), BAR Int. Ser. 102, II, Oxford, 79-89.

SAGUÌ, L. (1998): Il deposito della Crypta Balbi: una testimonianza imprevedibile sulla Roma del VII secolo?, Ceramica in Italia: VI-VII secolo. Atti del Convegno in onore di John W. Hayes (L. Saguì, ed.), Biblioteca di Archeologia medievale 14, Florencia, 305-330.

SALVIAT, F.; TCHERNIA, A. (2013): Vins, vignerons et buveurs de l'Antiquité, Roma.

VAPUR, Ö. (2009): Menderes Magnesiası Hypokaustlu Yapı Yerel Üretim Seramikleri, Diss. Ankara Üniversitesi.

VIZCAÍNO, J. (2009): La presencia bizantina en Hispania (siglos VI-VII), La documentación arqueológica, Antigüedad y cristianismo. Monografías históricas sobre la Antigüedad Tardía XXIV, Murcia.

VROOM, J. (2003): After Antiquity. Ceramics and Society in the Aegean from the 7 th to the 20th century A.C. A case study from Boetia, central Greece, Leiden.

VROOM, J. (2005): New Light on "Dark Age" pottery: A note on finds from Routh-western Turkey, Rei Cretariae Romanae Fautorum, Acta 39, Abingdon, 249-255.

VROOM, J. (2011): The other Dark ages: early medieval pottery finds in the Aegean as an archaeological challenge, When did Antiquity End? Archaeological case studies in three continents (R. Attoui ed.), BAR. Int. Ser. 2268, Oxford, 137-158.

WHITEHOUSE, D.; BARKER, G.; REECE, R.; REESE, D. (1982) The Schola Praeconum I, BSR 50, 53-101.

WHITEHOUSE, D.; COSTANTINI, L.; GUIDOBALDI, F.; PASSI, S.; PENSABENE, F.; PRATT, S.; REECE, R.; REESE, D. (1985): The Schola Praeconum II, BSR 53, 163-210. DOI: https://doi.org/10.1017/s0068246200011533

YANGAKI,A. G. (2004-2005): Amphores crétoises: le cas d'Éleutherna en Créte, $B C H$ 128/129, 1, 503-523. 